\title{
Checkpoint inhibitor develops histological autoimmune pancreatitis like type I diabetes. A case report
}

\begin{abstract}
Background: Immune checkpoint inhibitors are new cancer drugs that act stimulating immune adapted response of patient to obtain tumor regression. Immunotherapy can generate immune-related adverse events involved all organs, in particular checkpoint inhibitors PD1/PDL1 can develop autoimmune type 1 diabetes. CASE

Presentation: A 64 years old man affected by metastatic non-small cell lung carcinoma, after conventional therapy, was treated with pembrolizumab (a PD1/PDL1 checkpoint inhibitor): after ten weeks, he showed hyperglycemia with ketosis, stable reduced c-peptide, positive anti-glutamic acid decarboxylase antibodies therefore he was treated with insulin injection. The histological image of pancreas appeared like an immune-mediated insulitis.
\end{abstract}

Conclusion: New guidelines are recently published to inform clinicians and patients about frequent immune adverse events of these new immunotherapies.
Volume 10 Issue 3 - 2020

\author{
Mazzucato M,' Garelli S,' Betterle C,' \\ Presotto $F^{3}$ De Riva $C^{\prime}$ \\ 'Malattie Endocrine, del Ricambio e della Nutrizione - Ospedale \\ dell'Angelo, Italy \\ ${ }^{2}$ Endocrinologia, Univeristà di Padova, Italy \\ ${ }^{3}$ Medicina Interna, Ospedale dell'Angelo, Italy
}

Correspondence: Carlo de riva, Mestre Hospital,ASL3 Serenissima, Via paccagnella II, 30 I74 Mestre (VE), Italy, Email carlo.deria@aulss3.veneto.it

Received: June 03, 2020 | Published: June 17, 2020

Keywords: immunotherapies, insulitis, pathophysiological

\section{Case report}

Recently new cancer immunotherapies have revolutionized cancer treatment ${ }^{1}$ and their use is constantly increasing. Immune checkpoints are signal pathways that regulate the activation of Tcells in order to protect the tissues from damage triggered by pathogens and to prevent autoimmunity. Immune checkpoint inhibitors act on immune regulation and immune system checkpoint: the most famous are cytotoxic T-lymphocyte antigen 4 (CLTLA4) ${ }^{2}$ and programmed cell death protein 1 (PD1) and his ligands (PDL1 and PDL2). ${ }^{3}$ The target of these new therapies is to develop specificity and long-term memory of immune adapted response to achieve tumor regression. In comparison with conventional chemotherapies, these molecules play a role by stimulating immune system and not killing cancer cells so they have a longer latent time to start acting on cell mass (about 1620 weeks) but improve long-term overall survival. ${ }^{4,5}$ Immunotherapy induces immune-related adverse events which can involve all organ system $^{6,7}$ also the endocrine system. CTL4-targeted immunotherapy is associated with increased susceptibility to hypophysits and primary thyroid dysfunction, PD1-targeted immunotherapy is associated with primary thyroid disfunction and type 1 diabetes. ${ }^{8}$

In our medical clinic we visited a patient of 64 years old for hyperglycemia. He had an irrelevant remote history and one first relative affected by type 2 diabetes; the patient and nobody in his family had any autoimmune disease. Nine months earlier he had been diagnosed with non-small cell lung carcinoma with already evidence of repetitive nodal and vertebral disease (L1-L2). Oncologist started with conventional therapy with cisplatin but patient showed poor response and the disease progressed. The patient was candidate for treatment with Pembrolizumab. This therapy was started with absence of mutations for Epidermal Growth Factor Receptor (EGFR) and with presence of PD L1 expression in $26 \%$ of tumoral cell (PD-L1 IHC
28-8 Dako). Ten weeks after the beginning of treatment with PD1/ PDL1 checkpoint inhibitor compared hyperglycemia $(234 \mathrm{mg} / \mathrm{dl})$ with ketosis and mild increase of lipase (333U/L, normal value 140-200U/ L) in the absence of suggestive radiological pancreatitis. At the same time basal C-Peptide was severely reduced $(0.2 \mathrm{ng} / \mathrm{ml}$, normal value 0.78-1.89ng/ml); anti-glutamic acid decarboxylase antibodies were positive $(299 \mathrm{U} / \mathrm{ml}$, normal value $0-5 \mathrm{U} / \mathrm{ml})$, anti-insula antibodies were negative. Anti-IA2 (tyrosine phosphatase), antiinsulin or antiZnT8 antibodies are not performed and HLA haplotype wasn't tested. Multiple daily insulin treatment was started getting a discreet glycemic control. In the following weeks no recovery of basal betacell function (evaluated with $\mathrm{C}$ peptide assays) was detected.

Patient died for cardiac tamponade from metastatic hemopericardium, after 36 months from the oncological diagnosis. It followed an autoptic investigation: the pancreatic histology was indicative for typical insulitis and pancreas beta-cells expressed PDL1 in $42 \%$ of specific endocrine tissue (Figure 1-2).

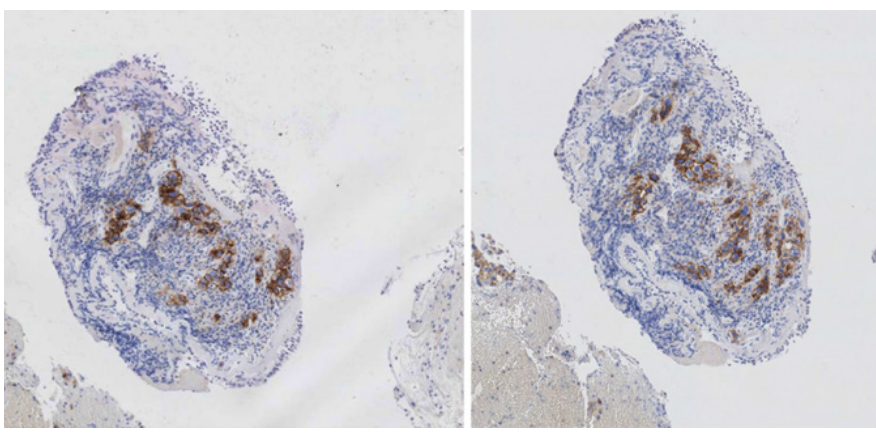

Figure I Immunohistochemistry showed the expression of PD-LI (clones used $22 \mathrm{C} 3$ and 28.8 ) with $26 \%$ positivity on a pulmonary biopsy specimen. 


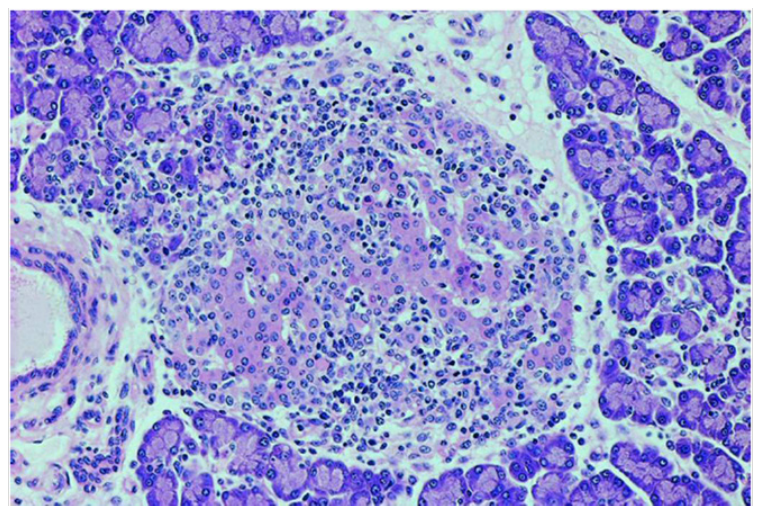

Figure $\mathbf{2}$ The pancreatic islets were infiltrated by numerous lymphocytes like a typical picture of immune-mediated insulitis.

The case described is a new form of autoimmune insulindependent diabetes, which appear rapidly as a consequence of the use of checkpoint inhibitors. Yoneda et al showed with immune histochemical analyses the number (more than 15 times higher than that of the control group) and the composition (CD8>CD4) of infiltrating CD3-positive $\mathrm{T}$ lymphocytes into pancreatic islets and exocrine region in the pancreas of a patient who developed type 1 diabetes after immune checkpoint inhibitor therapy. ${ }^{9}$ Non-obese diabetic mice lacking PD-L1 develop rapid onset of diabetes: in pancreatic islets low expression of PD-L1 is associated with increased $\beta$-cell injury. ${ }^{10}$ In patients affected by autoimmune type 1 diabetes, a decrease PD1 expression is reported also in peripheral CD4+ T cells. ${ }^{11}$

We show a histologic image of a typical insulitis related to use of a checkpoint inhibitor like the insulitis in autoimmune type 1 diabetes. The expression of PD-L1 on beta pancreatic cells may be implicated in immunological tolerance and may induce an autoimmune process. Other cases of type 1 diabetes related to PD1/PDL1 checkpoint inhibitors are described in literature. ${ }^{12,13}$ The increasing use of these new drugs stimulates the publication of new guidelines for the identification and treatment of specific side effects. ${ }^{14}$ These side effects may compare in 7-11 weeks after the beginning of therapies with checkpoint inhibitors, but they also may develop after months or years, and may have a long duration. It's important to identify these symptoms because the treatment is completely different from the conventional chemotherapy. In conclusion, immune checkpoint inhibitors (anti-CTLA-4 and anti-PDL-1) can induce the development of autoimmune diseases, among which autoimmune endocrinopathies (thyroiditis, adrenalite, hypophysitis, type 1 diabetes): therefore, before the beginning of the treatment, it's important to take an accurate personal and familiar history, to control hormones and glycemia before, during and after treatment and, if possible, to valuate a screening antibodies panel in order to select patients at greater risk of developing an autoimmune diseases.

\section{Conflicts of interest}

The authors have no conflicts of interest to declare.

\section{Funding}

None.

\section{Acknowledgments}

None.

\section{References}

1. Borghaei H, Smith MR, Campbell KS. Immunotherapy of cancer. Eur $J$ Pharmacol. 2009;625(1-3):41-54.

2. Pardoll DM. The blockade of immune checkpoints in cancer immunotherapy. Nat Rev Cancer. 2012;12(4):252-64.

3. Terme M, Ullrich E. IL-18 induces PD-1-dependent immunosuppression in cancer. Cancer Res. 2011;71(16):5393-5399.

4. Shih K, Arkenau HT, Infante JR. Clinical impact of checkpoint inhibitor as novel cancer therapies. Drugs. 2014;74(17):1993-2013.

5. Kannan R, Madden K, Andrews S. Primer on immuno-oncology and immune response. Clin J Oncol Nurs. 2014;18(3):311-317.

6. Champiat S, Lambotte $\mathrm{O}$, et al. Management of immune checkpoint blockade dysimmune toxicities: a collaborative position paper. Ann Oncol. 2016; 27(4):559-574.

7. Dine J, Gordon R, et al. Immune Checkpoint Inhibitors: an Innovation in Immunotherapy for the Treatment and Management of Patients with Cancer. Asia Pac J Oncol Nurs. 2017;4(2):127-135.

8. Byun DJ, Wolchok JD, Rosenberg LM, et al. Cancer immunotherapy immune checkpointblockade and associated endocrinopathies. Nat Rev Endocrinol. 2017;13(4):195-207.

9. Yoneda S, Imagawa A, Shimomura I. T-Lymphocyte Infiltration to Islets in the Pancreas of a Patient Who Developed Type 1 Diabetes After Administration of Immune Checkpoint Inhibitors. Diabetes Care. 2019;42(7):e116-e118.

10. Keir ME, Liang SC, Guleria I, et al. Tissue expression of PD-L1 mediates peripheral T cell tolerance. J Exp Med. 2006; 203(4):883-895.

11. Fujisawa R, Haseda F, Tsutsumi C, et al. Low programmed cell death-1 (PD-expression in peripheral CD4(+) T cells in Japanese patients with autoimmune type 1 diabetes. Clin Exp Immunol. 2015;180(3):452-457.

12. Perdigoto AL, Quandt Z, Anderson M, et al. Checkpoint inhibitor-induced insulindependent diabetes: an emerging syndrome. Lancet Diabetes Endocrinol. 2019;7(6):421-423.

13. de Filette JMK, Pen JJ, Decoster L, et al. Immune checkpoint inhibitors and type 1 diabetes mellitus: a case report and systematic review. Eur $J$ Endocrinol. 2019;181(3):363-374.

14. Thompson JA, Schneider BJ, Brahmer, et al. Management of Immunotherapy- Related Toxicities, Version 1.2019. J Natl Compr Canc Netw. 2019;17(3):255-289. 